\title{
ON THE RANDOM DISORIENTATION OF TWO CUBES
}

\author{
D. C. HANDSCOMB
}

1. Introduction. We are given two identical symmetrical bodies (e.g., cubes) with independent random orientations; then we can always, in several ways, turn one of these bodies about some axis through its centre of gravity, so as to bring it into the same orientation as the other body. The smallest angle of rotation needed will be called the disorientation, $d$, of the two bodies, and we shall be concerned with the distribution of $d$ under these conditions.

Ignoring symmetry, the relative orientation of the bodies is given uniquely (modulo rotations of $2 \pi$ ) by a single rotation; the required smallest rotation is the combination of this with some member of the symmetry group of the body. Using this fact only, and constructing random orthogonal matrices to describe the rotations, Mackenzie and Thomson (6) get an estimate of the distribution of $d$ for cubes, by the Monte Carlo method. I shall now show how, by another method, the distribution can be found explicitly.

2. Representation of rotations. We want a rotation to stand for the relative orientation of two independently oriented bodies; the distribution of rotations must therefore be invariant under any further arbitrary rotation of either body. Delthiel (5, pp. 99-106) sets out to obtain a distribution invariant in just such a manner. In the course of his work he represents the rotation $\mathscr{R}$ through angle $V$ about the axis with direction cosines $\alpha, \beta, \gamma$ by the coordinates $\lambda=\alpha \sin \frac{1}{2} V, \mu=\beta \sin \frac{1}{2} V, \nu=\gamma \sin \frac{1}{2} V, \rho=\cos \frac{1}{2} V$, satisfying $\lambda^{2}+\mu^{2}+\nu^{2}+\rho^{2}=1$.

He goes on to find the law of composition of rotations so represented, which is (altering his notation slightly):

$$
\begin{aligned}
& \lambda^{\prime \prime}=\lambda \rho^{\prime}+\mu \nu^{\prime}-\nu \mu^{\prime}+\rho \lambda^{\prime}, \\
& \mu^{\prime \prime}=-\lambda \nu^{\prime}+\mu \rho^{\prime}+\nu \lambda^{\prime}+\rho \mu^{\prime}, \\
& \nu^{\prime \prime}=\lambda \mu^{\prime}-\mu \lambda^{\prime}+\nu \rho^{\prime}+\rho \nu^{\prime}, \\
& \rho^{\prime \prime}=-\lambda \lambda^{\prime}-\mu \mu^{\prime}-\nu \nu^{\prime}+\rho \rho^{\prime},
\end{aligned}
$$

where the rotation $\mathscr{R}^{\prime \prime}$ is the resultant of $\mathscr{R}$ and $\mathscr{R}^{\prime}$ in that order. Note that $\lambda, \mu, \nu, \rho$ and $-\lambda,-\mu,-\nu,-\rho$ both correspond to the same rotation, a point which he does not mention. After this he changes his co-ordinates again and obtains the probability measure he is looking for.

However the equation $\lambda^{2}+\mu^{2}+\nu^{2}+\rho^{2}=1$ above may be regarded as the equation of a hypersphere in 4 -space, simply by taking $\lambda, \mu, \nu, \rho$ as Cartesian co-ordinates, so that every rotation corresponds to a pair of antipodal

Received April 30, 1957. 
points of the unit hypersphere. It is easy to verify that his measure is equivalent to the measure of hypersurface of this hypersphere; indeed its invariance under the group of rotations is evident from the law of composition, which shows that the transformation $\left(\lambda^{\prime}, \mu^{\prime}, \nu^{\prime}, \rho^{\prime}\right) \rightarrow\left(\lambda^{\prime \prime}, \mu^{\prime \prime}, \nu^{\prime \prime}, \rho^{\prime \prime}\right)$ is simply a rotation of 4 -space when $\lambda^{2}+\mu^{2}+\nu^{2}+\rho^{2}=1$.

The identity $\mathscr{I}$ corresponds to $\pm(0,0,0,1)$ and $\overline{\mathscr{R}}$, the inverse of $\mathscr{R}$, to $\pm(\lambda, \mu, \nu,-\rho)$.

Suppose now that we apply an arbitrary rotation $\mathscr{R}$ to a symmetrical body; what is the smallest rotation needed to restore it to its original aspect? If $G$ is the rotational symmetry group of the body in its original state, we want to find the rotation $\mathscr{S} \in G$, such that $\mathscr{R}^{\prime \prime}=\mathscr{S} \mathscr{R}$ has the smallest possible value of $V^{\prime \prime}$. Now the fourth line of the law of combination states that $\cos \frac{1}{2} V^{\prime \prime}=R \cdot \bar{S}$ (to use vector notation), and $\mathscr{S} \in G$ implies that $\overline{\mathscr{S}} \in G$, so that we need only look for the $\mathscr{S}$ giving the largest value to R.S, which is the member $S$ of the set of points of the hypersphere representing $G$, which is nearest to the point $R$ representing $\mathscr{R}$; we can then find the value of $V^{\prime \prime}$, which is $d$.

3. Application to Cubes. Take lines parallel to the edges of one cube as axes; its 24 symmetry rotations are then represented by the following points and their antipodes:
Identity
$(0,0,0,1)$

Rotations of :

$$
\begin{array}{ll}
\pi \text { about }(1,0,0) \text { etc. } \quad & (1,0,0,0)(0,1,0,0)(0,0,1,0) \\
\pm \frac{2}{3} \pi \text { about }(1,1,1) \text { etc. } & \left(\frac{1}{2}, \frac{1}{2}, \frac{1}{2}, \frac{1}{2}\right)\left(-\frac{1}{2},-\frac{1}{2},-\frac{1}{2}, \frac{1}{2}\right) \\
& \left(-\frac{1}{2}, \frac{1}{2}, \frac{1}{2}, \frac{1}{2}\right)\left(\frac{1}{2},-\frac{1}{2},-\frac{1}{2}, \frac{1}{2}\right) \\
& \left(\frac{1}{2},-\frac{1}{2}, \frac{1}{2}, \frac{1}{2}\right)\left(-\frac{1}{2}, \frac{1}{2},-\frac{1}{2}, \frac{1}{2}\right) \\
& \left(\frac{1}{2}, \frac{1}{2},-\frac{1}{2}, \frac{1}{2}\right)\left(-\frac{1}{2},-\frac{1}{2}, \frac{1}{2}, \frac{1}{2}\right) \\
& \left(0, \sqrt{ } \frac{1}{2}, \sqrt{ } \frac{1}{2}, 0\right)\left(\sqrt{ } \frac{1}{2}, 0, \sqrt{ } \frac{1}{2}, 0\right)\left(\sqrt{ } \frac{1}{2}, \sqrt{ } \frac{1}{2}, 0,0\right) \\
\pi \text { about }(0,1,1) \text { etc. } \quad\left(0, \sqrt{ } \frac{1}{2},-\sqrt{ } \frac{1}{2}, 0\right)\left(\sqrt{ } \frac{1}{2}, 0-\sqrt{ } \frac{1}{2}, 0\right)\left(\sqrt{ } \frac{1}{2},-\sqrt{ } \frac{1}{2}, 0,0\right)
\end{array}
$$$$
\pm \frac{1}{2} \pi \text { about }(1,0,0) \text { etc. }\left(\sqrt{ } \frac{1}{2}, 0,0, \sqrt{ } \frac{1}{2}\right)\left(0, \sqrt{ } \frac{1}{2}, 0, \sqrt{ } \frac{1}{2}\right)\left(0,0, \sqrt{ } \frac{1}{2}, \sqrt{ } \frac{1}{2}\right)
$$$$
\left(-\sqrt{ } \frac{1}{2}, 0,0, \sqrt{ } \frac{1}{2}\right)\left(0,-\sqrt{ } \frac{1}{2}, 0, \sqrt{ } \frac{1}{2}\right)\left(0,0,-\sqrt{ } \frac{1}{2}, \sqrt{ } \frac{1}{2}\right)
$$

Now the first 12 of these points, with their antipodes, are the vertices of a 24-cell, a regular polytope with Schläfli symbol $\{3,4,3\}$ (3, p. 156); the remainder are the vertices of a reciprocal polytope, also a 24 -cell, of the same size. This configuration is obtained directly from the cubic group by various methods by Coxeter $(2 ; 4)$ and by Robinson $(7)$. It is symmetrical, in the sense that all its vertices are equivalent. Since we want to work out a distribution involving only the vertex nearest to a random point of the sphere, we can with justice select any one vertex, say $(1,0,0,0)$, and let the random point be taken only from the sector of the hypersphere which is nearer to this vertex than to any other of the 48 . 
4. Calculation of the Distribution. We can now find the probability density for any angle $d$. The points of 4 -space subtending with the point $(1,0,0,0)$ an angle of $\frac{1}{2} d$ at the origin lie in the hyperplane $\lambda=\cos \frac{1}{2} d$, which meets the hypersphere in the sphere $\mu^{2}+\nu^{2}+\rho^{2}=\sin ^{2} \frac{1}{2} d$. Those points which subtend a smaller angle with this vertex than with any other of the 48 lie in the hypersolid bounded by the hyperplanes $\lambda \pm \mu=\sqrt{ } 2 \lambda$, $\lambda \pm \nu=\sqrt{ } 2 \lambda, \lambda \pm \rho=\sqrt{ } 2 \lambda, \lambda \pm \mu \pm \nu \pm \rho=0$, which are the loci of points equidistant from $(1,0,0,0)$ and from $\left(\sqrt{ } \frac{1}{2}, \pm \sqrt{ } \frac{1}{2}, 0,0\right),\left(\sqrt{ } \frac{1}{2}, 0, \pm \sqrt{ } \frac{1}{2}, 0\right)$, $\left(\sqrt{ } \frac{1}{2}, 0,0, \pm \sqrt{ } \frac{1}{2}\right),\left(\frac{1}{2}, \pm \frac{1}{2}, \pm \frac{1}{2}, \pm \frac{1}{2}\right)$, respectively. These hyperplanes meet $\lambda=\cos \frac{1}{2} d$ in the 6 planes of a cube: $\mu, \nu, \rho= \pm(\sqrt{ } 2-1) \cos \frac{1}{2} d$, and the 8 planes of an octahedron: $\pm \mu \pm \nu \pm \rho=\cos \frac{1}{2} d$, both concentric with the sphere. These 14 planes together bound a truncated cube, whose faces are 6 regular octagons and 8 equilateral triangles. (See (1), Plate I, No. 15, for illustration.)

If $d<45^{\circ}$, the whole sphere lies within the truncated cube, so that $p(d)$ is 48 times the probability density of points of 4 -space lying on this hypersphere, namely,

$$
\frac{48 \cdot \frac{1}{2} \cdot 4 \pi \cdot \sin ^{2} \frac{1}{2} d}{2 \pi^{2}} \cdot \frac{\pi}{180},
$$

where $d$ is measured in degrees. Thus if $d \leqslant 45^{\circ}$,

$$
p(d)=(2 / 15)(1-\cos d) .
$$

If $d>45^{\circ}$, we have to reduce this by a factor equal to the proportion of the surface of the sphere lying outside the truncated cube. If $45^{\circ}<d<60^{\circ}$, the sphere meets the octagonal faces only. The proportion of area cut off by these 6 planes is $3\left\{1-(\sqrt{ } 2-1) \cot \frac{1}{2} d\right\}$. Therefore if $45^{\circ} \leqslant d \leqslant 60^{\circ}$ :

$$
\begin{aligned}
p(d) & =(2 / 15)(1-\cos d)\left(3(\sqrt{ } 2-1) \cot \frac{1}{2} d-2\right) \\
& =(2 / 15)(3(\sqrt{ } 2-1) \sin d-2(1-\cos d)) .
\end{aligned}
$$

If $d>60^{\circ}$, the sphere meets the triangular faces also; it does not meet the edges provided that $\tan \frac{1}{2} d<2-\sqrt{ } 2$, or $d<60.6^{\circ}$. The proportion of area cut off by these 8 faces is $4\left(1-(1 / \sqrt{ } 3) \cot \frac{1}{2} d\right)$. Therefore if $60^{\circ}<d<60.6^{\circ}$ :

$$
p(d)=(2 / 15)(\{3(\sqrt{ } 2-1)+(4 / \sqrt{ } 3)\} \sin d-6(1-\cos d)) .
$$

If $d>60.6^{\circ}$, we have to increase this to allow for the sectors of sphere we have cut off twice, where an edge of the truncated cube goes inside the sphere. The proportion of the surface of a sphere common to the interiors of two small circles on it, of angular radii $A, B$, whose centres subtend an angle $C$ at the centre, is:

$$
\begin{gathered}
S(A, B, C)=\frac{1}{2 \pi}\left[\arccos \left(\frac{\cos A \cos B-\cos C}{\sin A \sin B}\right)\right. \\
\left.-\cos A \arccos \left(\frac{\cos B-\cos C \cos A}{\sin C \sin A}\right)-\cos B \operatorname{arc} \cos \left(\frac{\cos A-\cos B \cos C}{\sin B \sin C}\right)\right] .
\end{gathered}
$$


Each octagonal face meets the sphere in a circle of radius

$$
a(d)=\arccos \left((\sqrt{ } 2-1) \cot \frac{1}{2} d\right),
$$

each triangular face in one of radius

$$
b(d)=\operatorname{arc} \cos \left((1 / \sqrt{ } 3) \cot \frac{1}{2} d\right),
$$

two octagonal faces meeting at right angles and a face of each type meeting at an angle $\pi-c$, where $\cos c=1 / \sqrt{ } 3$. The maximum value of $d$ is attained when all the vertices of the truncated cube lie on the sphere, when $\cos d=\frac{1}{4}(2 \sqrt{ } 2-1), d=62.8^{\circ}$. Therefore if $60.6^{\circ}<d<62.8^{\circ}$ :

$$
\begin{aligned}
p(d)= & (2 / 15)[\{3(\sqrt{ } 2-1)+(4 / \sqrt{ } 3)\} \sin d \\
& \left.+\left\{12 S\left(a, a, \frac{1}{2} \pi\right)+24 S(a, b, c)-6\right\}(1-\cos d)\right] .
\end{aligned}
$$

Substituting and simplifying:

$$
\begin{gathered}
p(d)=(2 / 15)[\{3(\sqrt{ } 2-1)+(4 / \sqrt{ } 3)\} \sin d-6(1-\cos d)] \\
+(8 / 5 \pi)(1-\cos d)\left\{\arccos \left(\frac{\cot ^{2} \frac{1}{2} d}{3+2 \sqrt{ } 2-\cot ^{2} \frac{1}{2} d}\right)\right. \\
\left.+\frac{1}{2} \arccos \left(\frac{\cot ^{2} \frac{1}{2} d-2 \sqrt{ } 2}{3-\cot ^{2} \frac{1}{2} d}\right)\right\} \\
-(8 / 5 \pi) \sin d\left\{2(\sqrt{ } 2-1) \operatorname{arc} \cos \left(\frac{(\sqrt{ } 2-1) \cot \frac{1}{2} d}{\left\{1-(\sqrt{ } 2-1)^{2} \cot ^{2} \frac{1}{2} d\right\}^{\frac{1}{2}}}\right)\right. \\
\left.+(1 / \sqrt{ } 3) \operatorname{arc} \cos \left(\frac{(\sqrt{ } 2-1)^{2} \cot \frac{1}{2} d}{\left(3-\cot ^{2} \frac{1}{2} d\right)^{\frac{1}{2}}}\right)\right\} .
\end{gathered}
$$

This completes the distribution. The mean works out to be $42.7^{\circ}$.

I wish to record my thanks to the United Kingdom Atomic Energy Authority for a financial grant, to Messrs Mackenzie and Thomson for bringing this problem to my notice through a private communication and for independently checking my formulae, to Mr. J. M. Hammersley for helpful criticism, and to Dr. Coxeter for further references.

\section{REFERENCES}

1. W. W. R. Ball, Mathematical Recreations and Essays (11th ed., London, 1939).

2. H. S. M. Coxeter, The binary polyhedral groups ..., Duke Math. J., 7 (1940), 367-379.

3. Regular Polytopes (London, 1948).

4. - Regular honeycombs in elliptic space, Proc. Lond. Math. Soc. (3), 4 (1954), 471-501.

5. R. Delthiel, Probabilités Géométriques, Tome 2, Fasc. 2 of E. Borel, Traité du Calcul des Probabilités... (Paris, 1926).

6. J. K. Mackenzie and M. J. Thomson, Some statistics associated with the random disorientation of cubes, Biometrika, 44 (1957), 205-210.

7. G. de B. Robinson, On the orthogonal groups in four dimensions, Proc. Camb. Phil. Soc., 27 (1931), 37-48.

\section{Christ Church,}

Oxford 